\title{
Coulisses
}

Revue de théâtre

\section{Au carrefour du sacré et du profane. La Petite Messe solennelle de Gioacchino Rossini}

Claire-Marie Mille, Charlotte Nessi et Rédaction

\section{OpenEdition}

\section{Journals}

Édition électronique

URL : http://journals.openedition.org/coulisses/1660

DOI : $10.4000 /$ coulisses. 1660

ISSN : 2546-9460

Éditeur

Presses universitaires de Franche-Comté

\section{Édition imprimée}

Date de publication : 1 février 1991

Pagination : 65-71

ISSN : 1150-594X

\section{Référence électronique}

Claire-Marie Mille, Charlotte Nessi et Rédaction, « Au carrefour du sacré et du profane. La Petite Messe solennelle de Gioacchino Rossini », Coulisses [En ligne], 3 | Hiver 1991, mis en ligne le 04 juillet 2017, consulté le 21 octobre 2019. URL : http://journals.openedition.org/coulisses/1660 ; DOI : 10.4000/ coulisses. 1660

Ce document a été généré automatiquement le 21 octobre 2019

Coulisses 


\section{Au carrefour du sacré et du profane. La Petite Messe solennelle de Gioacchino Rossini}

\section{Claire-Marie Mille, Charlotte Nessi et Rédaction}

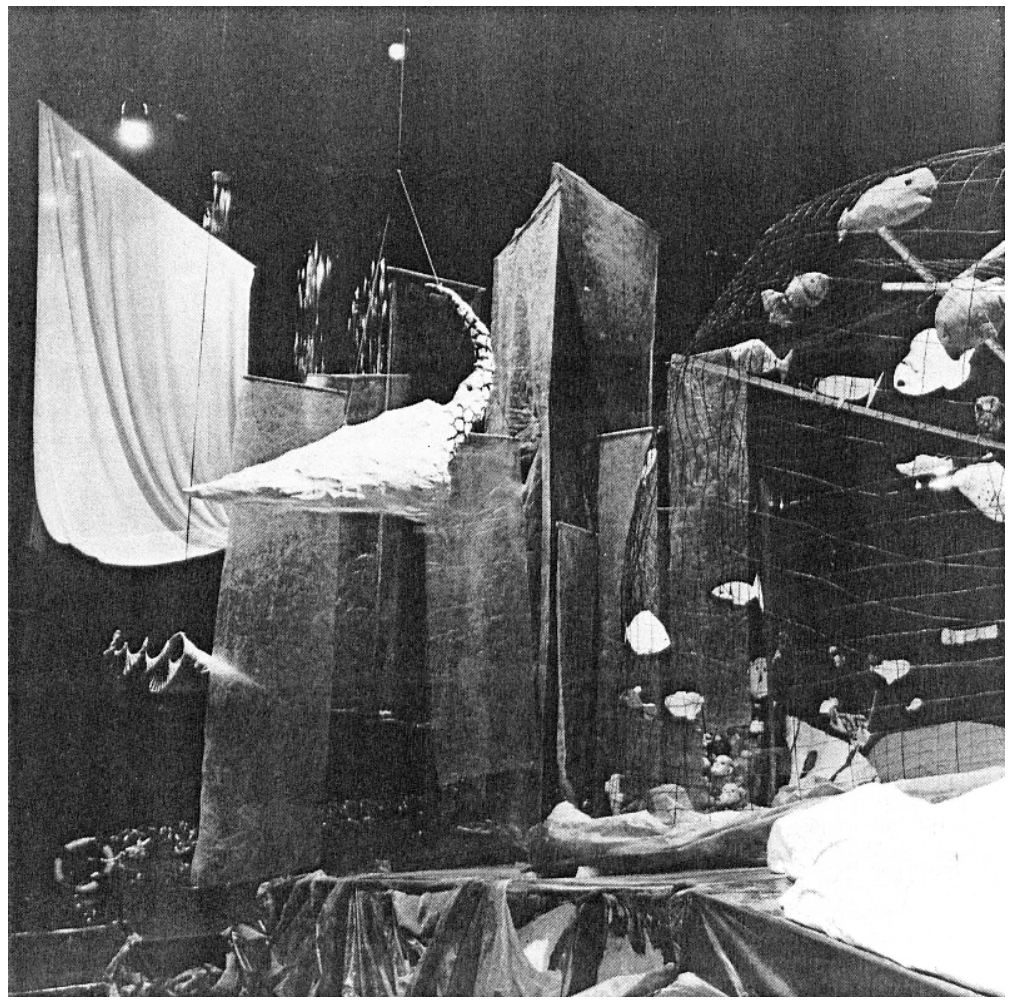

3 septembre 1990 - 20h30. Un monde fou pour cette première version scénique de la Petite Messe solennelle de Rossini, coproduction du Festival international de musique de Besançon, de l'ensemble Justiniana et du Centre polyphonique de Franche-Comté1. Le théâtre est plein, il faut rajouter des chaises en dépit du prix des places élevé. 
2 Un titre sybillin et passablement contradictoire: Petite Messe solennelle. Le décor confirme ce paradoxe. Un salon de musique, deux pianos, un harmonium, dans une verrière qui rappelle l'architecture de Baltard. Deux messieurs entrent en scène fort occupés à discuter de recettes au foie gras. La maîtresse de maison blonde et rose, vaque aux derniers préparatifs, arrange les bouquets. Les invités arrivent. Les robes des femmes aux couleurs vives, aux harmonies contrastées, les costumes des hommes ont de vague réminiscence avec les tableaux de Winterhalter. On prend place, les hôtes de ce salon se révèlent alors en être les artistes. Les deux pianistes, l'organiste, le chœur la basse, le ténor, l'alto, le soprano vont se donner réponse au cours d'une soirée dont le sujet est la messe.

3 Première partie: très ludique, reprend les rites de l'entrée avec le Kyrie et le Gloria. On repère quelques mesures ironiques, peut-être parodiques d'Offenbah et d'autocitation de La Traviata. On comprend mieux le titre La Petite Messe, une œuvre profane en quelque sorte. Peu à peu le silence se crée, non pas celui de la salle, qui est acquis, mais une espèce de silence intérieur, qui trouve son expression dans l'opposition entre l'éclatant Gloria et le très humain, "Domine deus, Agnus Dei, Filius patris, qui tollis peccata mundi, miserere nobis». Le sacré l'emporte sur le ludique, la méditation sur la conversation.

4 Deuxième partie: le salon s'est transformé en salle à manger. Une longue table, douze chaises. Le décor est planté pour la Cène. Les domestiques s'affairent à dresser le couvert. Le credo est affirmé, et sur les paroles « cruxifixus etiam pro nobis... et sepultus est ", on apporte un tableau représentant la crucifixion. Il est alors placé sur un chevalet et domine en quelque sorte le banquet. L'ironie reprend ses droits : la messe, l'art sacré, comme intermède esthétique.

Et exspecto resurrectionem mortuorum, et vitam venturi saeculi. Amen.

5 Les invités portent un toast, le domestique dispose une superbe pièce montée. Le sanctus peut commencer dans l'allégresse de la célébration eucharistique et/ou païenne, mais une femme se détache hiératique, sombre dans sa robe bleu profond, semblable à certains Velasquez. L'Agnus Dei, s'élève grave, profond, déchirant, alors qu'elle s'effondre lentement sur une chaise, puis sur la table avec le « dona nobis pacem ». La Petite Messe est devenue "solennelle ", au sein même de la fête mondaine, porteuse de l'interrogation poignante sur la destinée et la douleur.

6 Le spectateur reste perplexe. Il est peu banal de voir la messe traitée en spectacle, la messe comme opéra. Mais après réflexion, pourquoi pas ce miroir sur une de nos pratiques, où le fidèle, acteur à l'église puisque c'est lui qui répond, est appelé à contempler sa propre démarche. Alors que les signes cultuels et culturels l'invitent sans cesse à une réflexion ironique sur la pratique religieuse, la voix de l'alto l'emporte peu à peu vers cette méditation sur soi et sur sa condition. Sans doute on peut apprécier la représentation comme un pur plaisir musical, où le parti-pris du jeu sur l'ambiguité du titre est pleinement assumé. Le chef de chœur, Claire-Marie Mille, menue silhouette noire, sur fond coloré, âme de cette assemblée qu'elle tient sous sa baguette, avec la foi de l'œuvre à accomplir, est d'ailleurs le véritable officiant de cette Messe.

7 Messe ? Spectacle? Intermède esthétique? Méditation théologique et métaphysique? qui peut analyser la signification réelle du déferlement des applaudissements qui répondit à la conviction de tous les créateurs du spectacle? 


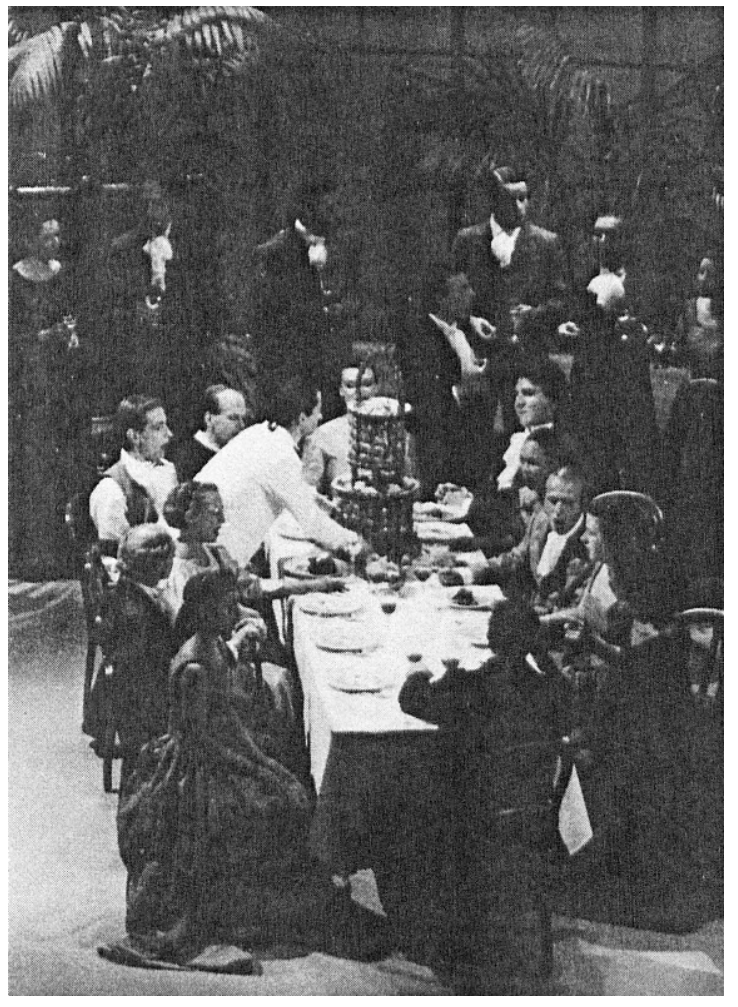

\section{À la sortie du spectacle, à chaud}

\section{Le grand public}

Réticences

- Ça m’a ennuyé...

- Je n'ai pas accroché... mais pourtant, c'est un bon spectacle...

- C'est lent... on attend qu'il se passe quelque chose et il ne se passe rien... Les déplacements du chœur et des acteurs restent très maladroits, leur jeu très artificiel.

- J'ai été déçue par la voix de la soprano.

- Comme d'habitude, au festival, il n'y avait qu'un public de notables.

\section{Compliments}

- J'ai beaucoup aimé... une excellente soirée, ce n'est pas si fréquent...

- J'ai été très surprise par la messe dans le salon! Quelle bonne idée !

- La voix de l'alto était superbe...

- Une surprise! La mise en scène est une découverte par rapport à la simple audition du disque... Je vais réécouter l'enregistrement...

- Des éclairages justes, qui n'écrasent ni la musique, ni les chanteurs... Du beau travail...

- Une soirée parfaite, avec de l'émotion et beaucoup d'humour.

Des personnalités de l'université, de la région, de la Direction des affaires culturelles de la ville de Besançon

- Un concert, le mot s'impose de louanges...

- Un exemple parfait de coproduction professionnels-amateurs...

- Un très grand moment, j'ai été comblé tant par la qualité de la mise en scène que 
par celles des vois...

- La qualité musicale et l'intensité dramatique étaient telles que j'ai fermé les yeux pour mieux écouter...

- Une mise en scène tout à fait respectueuse de l'esprit dans lequel Rossini a composé son œuvre...

- Une autre manière d'appréhender le sacré, avec gravité mais sans emphase.

\section{Entretiens} novatrices dans des domaines habituellement réservés aux hommes: la direction d'orchestre et la mise en scène d'opéra.

Le Centre polyphonique de Franche-Comté, organisme à vocation régionale créé en 1984, s'est donné pour mission de promouvoir une politique de développement de la pratique chorale. S'adressant à tous les chanteurs, débutants ou confirmés, il assure aussi bien la formation solfégique et vocale, l'éveil musical des enfants, que la formation du chef de chœur et le travail sur une œuvre particulière, un des éléments les plus importants de ses activités.

La sensibilisation à la musique contemporaine constituant un réel facteur d'enrichissement et de progrès de la chorale amateur, toutes les œuvres proposées et travaillées depuis 1986 appartiennent au répertoire choral du $\mathrm{xx}^{\mathrm{e}}$ siècle. La direction artistique du Centre est assurée par Claire-Marie Mille, premier prix de direction de chœur au CNMS de Lyon.

Est-ce la première fois que vous travaillez sur un opéra?

Claire-Marie Mille: Nous avons créé ensemble Charlotte Nessi et moi en avril 1989, à l'espace Planoise, Quichotte-Opéra Jazz, œuvre écrite par Mike Westbrook, livret de JeanLuc Lagarce.

Pourquoi la Petite Messe solennelle?

CMM : Cette œuvre nous tentait, parce que c'est une œuvre de Rossini peu connue à l'heure actuelle mais qui eut un certain succès à son époque comme l'attestent les critiques... et puis c'est une autre manière de présenter le sacré.

N'avez-vous pas craint que le spectacle soit ressenti comme provoquant?

CMM : Telle n'a pas été notre intention. L'œuvre comporte une progression et une profondeur qui englobent l'expérience de toute la vie humaine. Les mondanités, une certaine futilité en sont une des composantes, rendue par la parodie de la musique légère de l'époque. En revanche, n'importe quel prétexte peut donner lieu à un retour sur soi, et à une prise de conscience poignante de la vanité des choses humaines. La musique de Rossini est émouvante mais n'en est pas pour autant mystique.

Quel a été votre rôle dans la préparation du spectacle?

CMM : L'analyse musicale de l'œuvre et la direction musicale le chœur est composé d'amateurs qui chantent depuis trois, cinq ou six ans, mais c'était un énorme pari que de leur proposer de créer une œuvre dans le cadre du Festival de musique avec des chanteurs professionnels. Outre le niveau vocal, il fallait qu'ils devinssent des comédiens. Tous les choristes savaient qu'il s'engageaient dans une entreprise, exigeant d'eux une grande disponibilité, des répétitions mais aussi des stages de travail d'acteur. Les solistes ont été engagés après audition. Ils ont répété une semaine à Besançon avec l'ensemble du groupe ce qui est très rare dans le monde de l'opéra où les chanteurs arrivent la veille du spectacle; il est vrai qu'ils savent leur partition. 
Berlioz avec trente chorales de Franche-Comté, environ huit cents à mille choristes, des
solistes internationaux, l'orchestre de Toulouse, sous la direction de Michel Plasson. Créer une école maitrisienne à Dole, c'est-à-dire créer en France des chorales d'enfants à la manière des anglais.

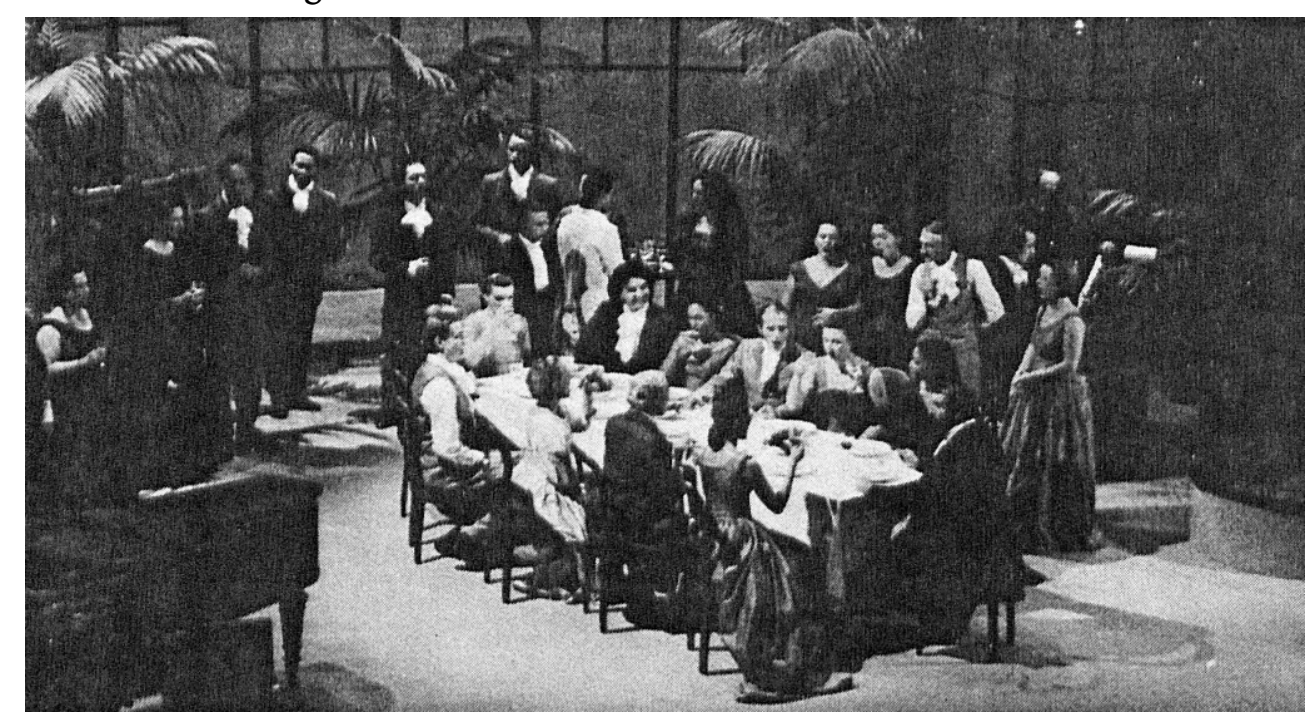

\section{L'ensemble Justiniana}

L'ensemble tire son nom du mont Justin, en Haute-Saône, où il a été créé en 1975 par des adolescents passionnés par la musique de chambre. Il s'est ensuite tourné vers l'opéra et se consacre à la recherche de nouvelles productions lyriques ; appréhension d'œuvres du répertoire ou production d'œuvres nouvelles ouvertes à différentes expressions musicales, sa démarche vise à diffuser le lyrique en dehors des « machinesopéra ", structures lourdes et coûteuses réservées à une élite, en utilisant des lieux de spectacles nouveaux, et sensibiliser à l'art lyrique un public non averti. Rompant avec les traditions du monde lyrique, l'ensemble Justiniana s'efforce d'intégrer des ensemble amateurs à une production lyrique professionnelle.

\section{Charlotte Nessi, Pourquoi la Petite Messe solennelle?}

Charlotte Nessi : C'est un projet que je porte depuis très longtemps en moi, depuis 1982, depuis la version concert. J'ai voulu essayer de rendre l'atmosphère de l'époque, cette réception mondaine $d u$ banquier commanditaire telle qu'elle est contée par les journalistes. J'ai aussi essayer d'imaginer ce qui a pu se passer dans la tête de Rossini. Quand à l'âge de soixante et onze ans, il compose sa petite messe solennelle, il a déjà écrit plus de quarante opéras qui ont assuré sa renommée, parmi lesquels Tancredi, L'Italienne à Alger, Le Barbier de Séville, La Cenerentola, Guillaume Tell. Il ironise d'ailleurs sur cette œuvre : «Hélas, le dernier péché mortel de ma vieillesse », et ailleurs : "Bon 
Dieu - la voilà terminée cette pauvre petite messe - Est-ce bien de la musique sacrée que je viens de faire ou bien de la sacrée musique? J'étais né pour l'opéra Buffa, tu le sais bien! Peu de science, un peu de cœur, tout est là. Sois donc béni, et accorde-moi le paradis. »

Rossini invente ainsi une forme théâtrale de la musique religieuse, réalisant la fusion du dramatique et du sacré.

Pensée dans le cadre d'une représentation, c'est-à-dire hors des murs de l'église, cette œuvre entend prouver que le sentiment religieux peut vivre en dehors des chemins conventionnels, qui, malheureusement, ne mènent, bien souvent, qu'au sectarisme et au fanatisme.

Quelle a été l'idée directrice de votre mise en scène?

$C N$ : Donner à voir la théâtralité de la musique tout en laissant la priorité à la musique.

Qu'est-ce qui vous a poussée à devenir en quelque sorte le metteur en scène de la musique?

$\mathrm{CN}$ : J'ai été formée au Conservatoire national de Strasbourg et je travaille pour une thèse de musicologie à Paris IV sur la mise en scène de Pelléas et Mélisande.

Pourquoi avoir choisi ce décor de verrière?

$C N$ : J'ai voulu rappeler à la fois l'époque, le scientisme, l'esthétique du Crystal Palace et la tour Eiffel, mais faire aussi référence à l'architecture religieuse, à la cathédrale.

Quelle a été votre méthode de travail ?

$C N$ : J'ai d'abord observé les personnalités de chacun des choristes de manière à pouvoir leur donner un personnage, puis nous avons eu un stage fondé sur l'improvisation sans chant et un travail individuel avec chacun. Il s'agit de la réalisation d'une équipe, ce qui est caractéristique du théâtre. Les costumes ont été pensés par François Tomsu de la même manière, en fonction de l'individualité et de la silhouette de chacun mais aussi par rapport à l'ensemble.

Quels sont vos projets?

$\mathrm{CN}$ : Je travaille actuellement à la mise en scène d'un opéra contemporain Beau soir et ciel d'orage de Gérard Pesson pour le Festival musical au théâtre Jean Vilar avec une trentaine de professionnels. En février, à l'opéra de Montpellier je présenterai Didon et Énée de Purcell avec trente-cinq jeunes filles et six solistes enfants.

Vous avez déjà travaillé avec des enfants?

$C N$ : Oui. En particulier Le Petit Ramoneur de Benjamin Britten en 1984 avec deux mille cinq cents enfants des écoles de Franche-Comté. Cette année je vais également créer à l'opéra Bastille, puis à Montbéliard, L'Arche de Noë de Britten avec trois cents enfants. Travailler avec des enfants est très troublant et très exigeant. Il faut se remettre constamment en question, être constamment à l'écoute. 


\section{NOTES}

1. Direction musicale: Claire-Marie Mille; mise en scène: Charlotte Nessi ; décor lumières : Gérard Champion; conception costumes : François Tomsu ; maquillage : Valérie Weiss. Solistes : soprano: Catherine Antonicelli; alto: Roseline Cyrille; ténor: Daniel Galvez Vallejo; basse : Daniel Lavall; chœur: 24 choristes du Centre polyphonique de Franche-Comté. Musiciens: piano I : André Litolff ; piano II : Yves Muller ; harmonium : André Larroque.

\section{AUTEURS}

\section{CLAIRE-MARIE MILLE}

Directrice du Centre polyphonique de Franche-Comté (10 rue Jean-Jacques-Rousseau, 25000 Besançon), chef de chœur.

\section{CHARLOTTE NESSI}

Directrice de l'ensemble Justiniana (Mont-Justin, 70100 Villersexel), metteur en scène. 